\title{
The Composition of Interplanetary Coronal Mass Ejections
}

\author{
Thomas H. Zurbuchen", L. A. Fisk ${ }^{*}$, S. T. Lepri ${ }^{*}$, and R. von Steiger ${ }^{\dagger}$ \\ - Department of Atmospheric, Oceanic and Space Sciences, University of Michigan, \\ 2455 Hayward St., Ann Arbor, MI 48109-2143, USA \\ ${ }^{\dagger}$ International Space Science Institute, Hallerstrasse 6, CH-3012 Bern, Switzerland
}

\begin{abstract}
Interplanetary coronal mass ejection (ICME) associated plasma can exhibit signatures in elemental, ionic and isotopic composition. These signatures occur in less than 50\% of all ICMEs, but are very indicative of ICME plasma. We review these compositional anomalies and briefly discuss a physical scenario that could be responsible for these anomalies.
\end{abstract}

\section{INTRODUCTION}

The interplanetary consequences of coronal mass ejections (CMEs) have been a central topic of heliospheric physics since their discovery in the 1970s. Research on Interplanetary Coronal Mass Ejections (ICMEs) has focused on two major aspects: the identification of and the physical drivers for ICME material.

First, the identification of ICME material has been the focus of many publications, e.g., by Richardson et al. $[1,2]$. Common plasma signatures of ICMEs in the solar wind include counterstreaming electrons, anomalously low proton and electron temperatures, strong magnetic fields, low plasma beta, and smooth field rotations [3]. These studies have stressed the diverse nature of ICME-associated plasmas and the resulting lack of a coherent set of signatures that can be identified as "necessary and sufficient" for the presence of ICME plasma.

Second, progress is being made in relating CME signatures to the underlying physical processes that govern CME initiation in the low corona, and propagation of CMEs from the corona into the heliosphere. In situ measurements provide crucial constraints for these CME models [4] and can serve as "ground-truth" measurements for various CME models. Even though substantial progress has been made, CME models are still not mature enough for some of these detailed tests [5].

Composition measurements of ICMEs provide important contributions for both research aspects discussed above. Before we summarize recent results, we would like to note that some valuable solar wind com- position data of ICMEs preceded modern dedicated composition experiments. A comprehensive description of such data can be found in a paper by Bame [6] summarizing his 1982 Solar Wind conference presentation.

In this paper, two major issues will be addressed using Ulysses and ACE composition data measured by each of their Solar Wind Ion Composition Spectrometers (SWICS) [7]. First, we will focus on the compositional signatures of average ICMEs. We specifically concentrate on the elemental composition of "driver gas" identified by an unusually high alpha-to-proton $\left(\mathrm{He}^{2+} / \mathrm{H}^{+}\right)$ratio. We will then propose a physical scenario that could produce these compositional measurements.

\section{COMPOSITIONAL SIGNATURES}

This section discusses the observed compositional anomalies of ICMEs. The diversity and geometrical complexity of ICMEs, based on in situ plasma and field signatures [3], become evident when looking at these compositional signatures. It is therefore very difficult to define average properties of this highly diverse plasma population. We now have access to 11 years of Ulysses data and 4 years of ACE data. From these data we know that there are two classes of relatively rare ICMEs that are fundamentally different than the vast majority of ejections observed by these instruments. First, there are unusually "cold events" that are characterized by a substantial fraction of all ele-

CP679, Solar Wind Ten: Proceedings of the Tenth International Solar Wind Conference,

edited by M. Velli, R. Bruno, and F. Malara

(C) 2003 American Institute of Physics 0-7354-0148-9/03/\$20.00 
ments with low charge states. One prominent example is discussed by Gloeckler et al. [8] based on ACESWICS data. These events show unusual fractionation patterns [9] uncharacteristic of the vast majority of ICMEs. Second, there are high-latitude events described by Gosling [10], which have a composition very similar to coronal-hole-associated wind. These two classes of ICMEs are of considerable interest but will not be discussed further in this paper because of their apparently insignificant relative contribution to ICMEs during the solar cycle.

The following subsections will summarize our present knowledge of compositional anomalies in the majority of the ICMEs (those that fall outside the two categories described above) observed by ACE and Ulysses.

\section{Unusual Ionic Composition}

It has been pointed out that a strong preference exists for ICMEs to have charge states indicative of unusually hot electron temperatures $[11,12,13]$. Figure 1 shows plasma and composition data of an ICME observed by the combined ACE plasma and field sensors. The figure shows a time period of 14 days during 1999 that contains ICME ejecta, the trailing edge of coronalhole-associated wind, and variable slow wind. The ionic composition during large parts of this time period is unusual. Both $\mathrm{O}$ and $\mathrm{Fe}$ show charge states that are clearly above the average solar wind composition. The

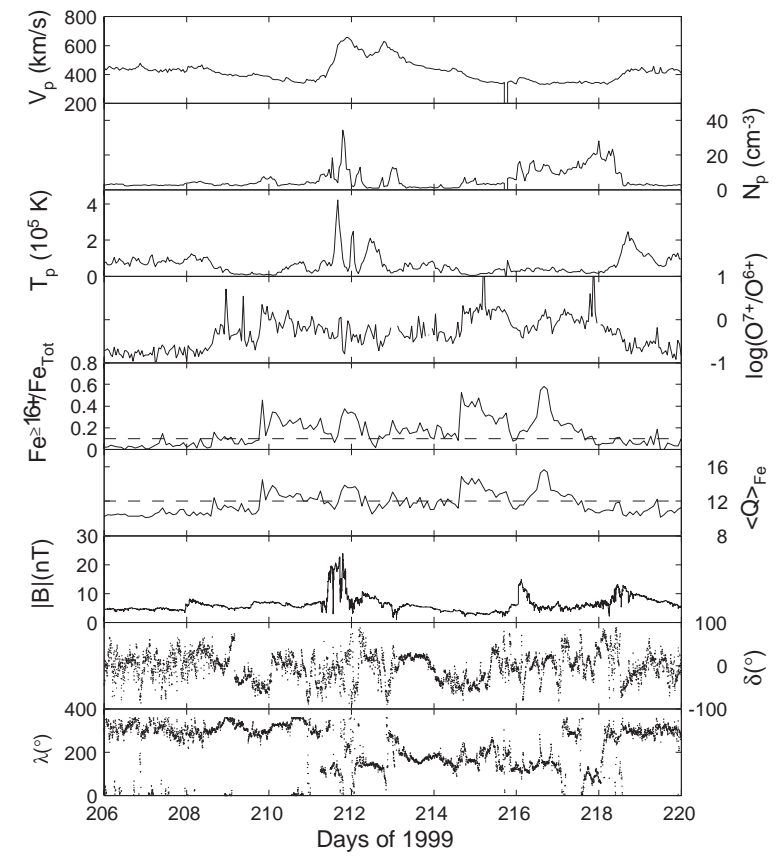

FIGURE 1. ICME event observed by ACE. The time period shows an elongated complex structure that exhibits compositional anomalies that are typical of $\sim 50 \%$ of all ICMEs. compositional signatures are highly time-dependent, indicating hotter and cooler parts in the ICME. In a comprehensive study by Lepri et al. [12], roughly 50\% of all ICMEs, as identified by a large set of plasma and field signatures, had long Fe charge state enhancements. Conversely, over $95 \%$ of all Fe charge state enhancements that extended over 20 hours were found to be ICMEs. Even though there are no similar studies on $\mathrm{O}$ charge states, similar behavior might be expected when studying $\mathrm{O}^{7+} / \mathrm{O}^{6+}[13]$.

The observation of predominantly hot charge states in ICMEs might be surprising. Remotely observed CMEs often have a three-part structure with a bright rim, indicative of the interaction region of $\mathrm{CME}$ plasma with the ambient solar wind, a dark cavity, and a bright high-density core that is presumably associated with an erupting prominence. Out of over 200 ICMEs observed at ACE, only $<2$ have very low Fe charge states. If cool prominence plasma ever makes it into space, it either has to undergo rapid ionization during the CME release, or it needs to be confined to a distinguishably small volume within the ICME. In the absence of any of these explanations, the observed small fraction of "cold" ICMEs is not consistent with any prominence plasma making it into the heliosphere.

\section{Unusual Elemental Composition}

One of the most prominent ICME signatures is the $\mathrm{He}^{2+} / \mathrm{H}^{+}$ratio [3]. Typically, $\mathrm{He}^{2+} / \mathrm{H}^{+}>0.08$ is considered unusually high and can be used for the identification of ICMEs from the Sun into the outer heliosphere [14] (does this mean you can observe high alpha to proton ratios at the sun and out to Ulysses? This isn't quite clear here). The $\mathrm{He}^{2+} / \mathrm{H}^{+}$ratio has been measured over many decades using electrostatic systems and Faraday cup systems $[15,16]$. Traditionally, periods of unusually large $\mathrm{He}^{2+} / \mathrm{H}^{+}$ratio have been identified as "driver gas" even before these data were put into context with CME observations at the Sun [6]. It is now clear that these signatures exist in only a fraction of the plasma associated with an ICME. The geometrical association of these periods with parts of the traditional three-part structure is unclear.

Using SWICS data, ICMEs can be studied using a more comprehensive set of data. Reinard et al. [13] examined elemental composition data from over 20 ICMEs in 1998-1999 identified using bi-directional electron signatures. She compared them with the composition of slow solar wind offset by 1-2 days from the ejecta. The result was that the average elemental composition of $\mathrm{Fe} / \mathrm{O}$ of ICMEs was indistinguishable from the slow solar wind composition. Several of the events in question had substantial $\mathrm{He} / \mathrm{H}$ enhancements accompanied by similar $\mathrm{He} / \mathrm{O}$ enhancements. The enhancements in He therefore seemed to occur without 


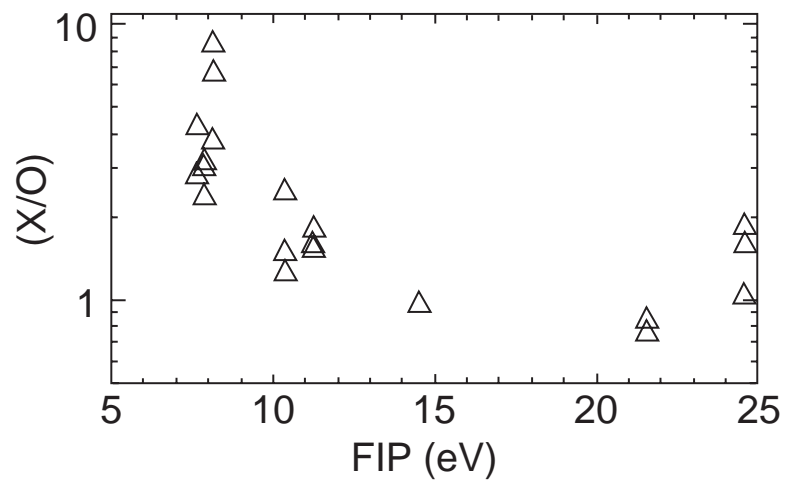

FIGURE 2. Elemental composition of driver gas during the Ulysses mission. The average composition is very similar to the composition of the solar wind with the exception of $\mathrm{He} / \mathrm{O} . \mathrm{Si} / \mathrm{O}$ is artificially enhanced due to "blending" with $\mathrm{Fe}$ of high charge state.

significantly affecting the $\mathrm{O}$ and $\mathrm{Fe}$ abundances relative to $\mathrm{H}$.

A more comprehensive test can be carried out using Ulysses-SWICS data. A search has been performed, over the entire Ulysses data set, for full days that have $\mathrm{He}^{2+} / \mathrm{H}^{+}>0.8$ in order to ensure sufficient statistical accuracy for all rare elements in question. Due to the intermittent nature of these enhancements, it is difficult to find those that span the entire day, except in a few cases. Figure 2 shows the elemental abundance of $\mathrm{Fe}$, $\mathrm{Mg}, \mathrm{Si}, \mathrm{S}, \mathrm{Ne}$, and $\mathrm{He}$ of this "driver gas" ordered by First Ionization Potential (FIP). Si is found to be contaminated by Fe of a very high charge state (perhaps $16+$ ), and therefore has an unusually high elemental abundance. All other elements have abundances that are very similar to the slow solar wind, fractionated according to the FIP. We therefore conclude that, even though ICMEs can exhibit order-of-magnitude enhancement of $\mathrm{He}^{2+} / \mathrm{H}^{+}$, the elemental abundances show little or no fractionation beyond the common FIP fractionation of the slow solar wind.

\section{Unusual Isotopic Composition}

Unusual enhancements of ${ }^{3} \mathrm{He} /{ }^{4} \mathrm{He}$ in ICMEs have been reported based on early plasma measurements [6]. These enhancements are very interesting because of the similar fractionation effect observed in Solar Energetic Particles (SEP) [16]. Figure 3 compares ${ }^{3} \mathrm{He} /{ }^{4} \mathrm{He}$ of slow, fast, and ICME-associated solar wind. Slow solar wind values are from Geiss [17] and Ogilvie et al. [18], fast solar wind values are from Gloeckler and Geiss [19] and the ICME values came from Gloeckler et al. [8] and Ho et al. [20]. We do not currently know how often these enhancements occur or how these signatures correlate with other ICME plasma and compositional signatures. There are no reports of isotopic frac-

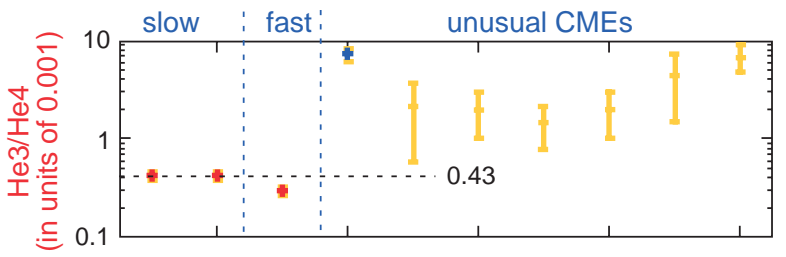

FIGURE 3. The He isotopic ratio in various solar wind regimes.

tionation in ICMEs of elements other than He that are above the detection limit.

\section{PHYSICAL SCENARIO}

This section discusses an interpretation of the compositional signatures of ICMEs in the context of the physical processes governing CMEs in the solar corona. The compositional signatures to be explained are fourfold:

a) FIP fractionation,

b) $\mathrm{He} / \mathrm{H}$ enhancements,

c) Unusually high charge states of heavy ions, and

d) ${ }^{3} \mathrm{He} /{ }^{4} \mathrm{He}$ enhancements.

The overall chemical composition of ICMEs is very similar to the composition of the slow solar wind, which is closely associated with coronal loops [21]. These loops are presumed to undergo successive reconnections in the vicinity of their footpoints, as sketched in Fig. 4a. These reconnection events lead to successive FIP fractionation, perhaps by a process not unlike the one described by Von Steiger and Geiss [22]. During this process, gravitational settling presumably also plays a role, most severely affecting $\mathrm{He} / \mathrm{H}$. Therefore, the depletion of $\mathrm{He}$ in these loops must be accompanied by an enhancement of $\mathrm{He} / \mathrm{H}$ in the vicinity of the loop footpoints.

When the magnetic structure of these loops becomes unstable, the erupting field will reconnect with the overlaying magnetic field-lines. This process will drive reconnection currents in these erupting loops, as sketched in Fig. 4b. These currents are mostly carried by supra-thermal electrons. The electrons have two primary effects: (1) they first ionize the ambient plasma, leading to heating and rapid ionization of heavy ions; and (2) they can also interact with the chromosphere, leading to chromospheric evaporation at the footpoints of the loops where $\mathrm{He} / \mathrm{H}$ is enhanced. The latter effect then gives rise to an excess of $\mathrm{He} / \mathrm{H}$ in the erupting loops. In a plasma that carries a current and has elevated $\mathrm{He} / \mathrm{H}$, wave-particle interactions with ${ }^{3} \mathrm{He}$ will lead to heating of ${ }^{3} \mathrm{He}[16]$. As a result, this heating will enhance the ${ }^{3} \mathrm{He} /{ }^{4} \mathrm{He}$ ratio. The eruption will therefore tend to produce all three compositional 
(a)

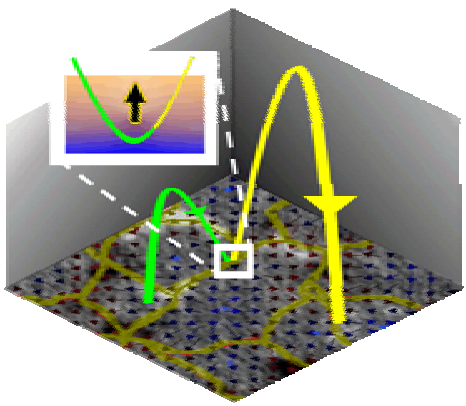

FIGURE 4. A physical scenario to explain the observed compositional signatures. Plasma is transported into loops through successive reconnection events, $\mathrm{He} / \mathrm{H}$ is depleted in these loops, enhancing $\mathrm{He} / \mathrm{H}$ below these loops to large values. During $\mathrm{CME}$ eruption, reconnection-driven currents lead to compositional changes in the loop plasma, and lead to chromospheric evaporation.

signatures that distinguish solar wind from ICME plasma.

\section{CONCLUSIONS}

Compositional signatures of ICMEs have been described, covering elemental, ionic, and isotopic composition anomalies. Even though the study of these signatures in situ has matured [2], these data have not been sufficiently analyzed in the context of the physical processes that govern CME eruptions. Physical scenarios such as described above are possible and need to be incorporated into a next-generation CME model therefore providing important constraints on the generation and eruption of CMEs and their propagation to Earth.

\section{REFERENCES}

1. Richardson, I. G., Cliver, E. W., and Cane, H. V., J. Geophys. Res. 28, 2569 (2000).

2. Richardson, I. G. et al., these proceedings, 2002.

3. Gosling, J. T., "Coronal Mass Ejections: An Overview," in Coronal Mass Ejections, edited by N. Crooker, J. A. Joselyn, and J. Feynman, Geophys. Monograph 99, 1997, p. 9.

4. Riley, P., Linker, J. A., and Mikic, Z., J. Geophys. Res. 106, 15889 (2001).

5. Manchester, W., et al., these proceedings, 2002.

6. Bame, S. J., "Solar Wind Minor Ions - Recent Observations," in Solar Wind 5 Proceedings, edited by M. Neugebauer, NASA Conference Proceedings 2280, 1983.
7. Gloeckler, G., et al., Astron. Astrophys. Suppl. Ser. 92, 267, 1992.

8. Gloeckler, G., et al., Geophys. Res. Lett. 26, 157 (1999).

9. Wurz, P., et al., these proceedings.

10. Gosling, J. T., Riley, P., McComas, D. J., and Pizzo, V. J., J. Geophys. Res. 103, 1941 (1998).

11. Henke, T., et al., Geophys. Res. Lett. 25, 3465 (1998).

12. Lepri, S. T., et al., J. Geophys. Res. 106, 21,231 (2001).

13. Reinard, A.A., et al., "Comparison between Average Charge States and Abundances of Ions in CMEs and the Slow Solar Wind," in SOHO/ACE workshop Solar and Galactic Composition, edited by R. F. WimmerSchweingruber, AIP Conference Proceedings 598, Melville, New York, 2001, p. 139.

14. Paularena, K. I., Wang, C., von Steiger, R., and Heber, B., Geophys. Res. Lett. 28, 2755 (2001).

15. Aellig, M. R., Lazarus, A. J., and Steinberg, J. T., Geophys. Res. Lett. 28, 2767 (2001).

15. Borrini, G., Gosling, J. T., Bame, S. J., and Feldman, W. D., Solar Physics 83, 367 (1983).

16. Fisk, L. A., Astrophys. J. 224, 1045 (1978).

17. Geiss, J., Eberhardt, P., Buehler, F., Meister, J., and Signer, P., J. Geophys. Res.,75, 5972 (1970).

18. Ogilvie, K. W., Coplan, M. A., Bochsler, P., and Geiss, J., J. Geophys. Res., 85, 6021 (1980).

19. Gloeckler, G., and Geiss, J., Space Sci. Rev. 84, 275 (1998).

20. Ho, G., Hamilton, D. C., Gloeckler, G., and Bochsler, P., Geophys. Res. Lett. 27, 309 (2000).

21. Zurbuchen, T. H., Fisk, L. A., Gloeckler, G., and Schwadron, N. A., Space Sci. Rev. 85, 397 (1998).

22. Von Steiger, R., and Geiss, J., Aston. Astrophys. 225, 222 (1989). 